\title{
Software Refactoring Technique for Code Clone Detection of Static and Dynamic Website
}

\author{
K. Kanagalakshmi. Ph.D. \\ Associate Professor \\ Department of Computer Application \\ Vidyasagar College of Arts and Science \\ Udumalpet - 642126 .
}

\author{
R.Suguna \\ Research Scholar \\ Department of Computer Application \\ Vidyasagar College of Arts and Science \\ Udumalpet - 642126
}

\begin{abstract}
Now-a-days cloning of codes or programs of the developer or authorized person leads a positive approach. But the code cloning is done by unauthorized person leads a negative approach. In the recent years, many clone detection tools have been proposed. It produces an over whelming volume of simple clones of data or structure [3]. Code clone detection the content similarity between the programs or webpages. An attempt is made to desgn a method called "SD Code Clone Detection" for both static and dynamic webpages. It is based on levenshtein's approach. This method comprises some steps like, parsing \& analysis, tree construction, code similarity measure and clone detection. Experiments are carried out with open source websites and webpages created by some volunteers. Experimental results are recorded and are showing the better detection rate.
\end{abstract}

\section{Keywords}

Refactoring, clone detection, code clone, static and dynamic pages, DOM tree construct, Levenshtein distance algorithm.

\section{INTRODUCTION}

Refactoring is a process of transforming the program without affecting the behavior and semantics and to improve the quality [24]. In other term code refactoring is the process of restructuring the existing computer code by changing the factors without affecting its external behavior [24]. The refactoring process also involves in the removal of duplication and simplification of unclear code[34]. The refactoring process offers many advantages such as improved code readability and reduced complexity to improve source code maintainability, creation of expressive internal structure [24]. The maintainability and extensibility are the two major benefits of refactoring. But the other side of code refactoring is called code clone. It is about the similarity of codes. Code clone can be defined as a similar program or code structure of considerable size and significant similarity [1]. Section 2 provides the literature review. In section 3 the proposed methods is discussed. Experimental results are recorded in section 4 and section 5 concludes the work.

\section{LITERATURE REVIEW}

The literature survey shows that cloning is an active area of research [1]. Many refactoring and clone detection tools and approaches have been proposed. A literature survey has been made to have a knowledge on code clone detection and its techniques[3]. Daniel. B [5] proposed a techniques and described some examples of refactoring such as renaming program element to be better convey its meaning, replacing field references splitting large classes etc., many other code refactor techniques have been proposed for code or software systems $[2,4,6,7,8,9,10,15$, $16,17,11,12,20,13,14,19,28,22,24,25,26,27,28,29,30,31,32$, 33].

\section{PROPOSED METHOD: SD CODE CLONE DETECTION TECHNIQUE}

A approach to clone mining for Web applications has been proposed together with a prototype implementation for dynamic web pages. The proposed methods analyze the page structure, implemented by specific sequences of HTML tags, and the content displayed for both dynamic and static pages. Moreover, for a pair of dynamic web pages we also consider the similarity degree of their source is considered. The similarity degree can be adapted and tuned in a simple way for different web applications in one- to- many. The proposed method called "SD Code Clone detection technique (SDCC) aims the detection of clones on both static and dynamic web pages. The proposed model consists of 4 phases namely content feeding, parsing and analysis refactoring (code extraction, DOM tree and similarity calculation), clone deduction as shown in fig.

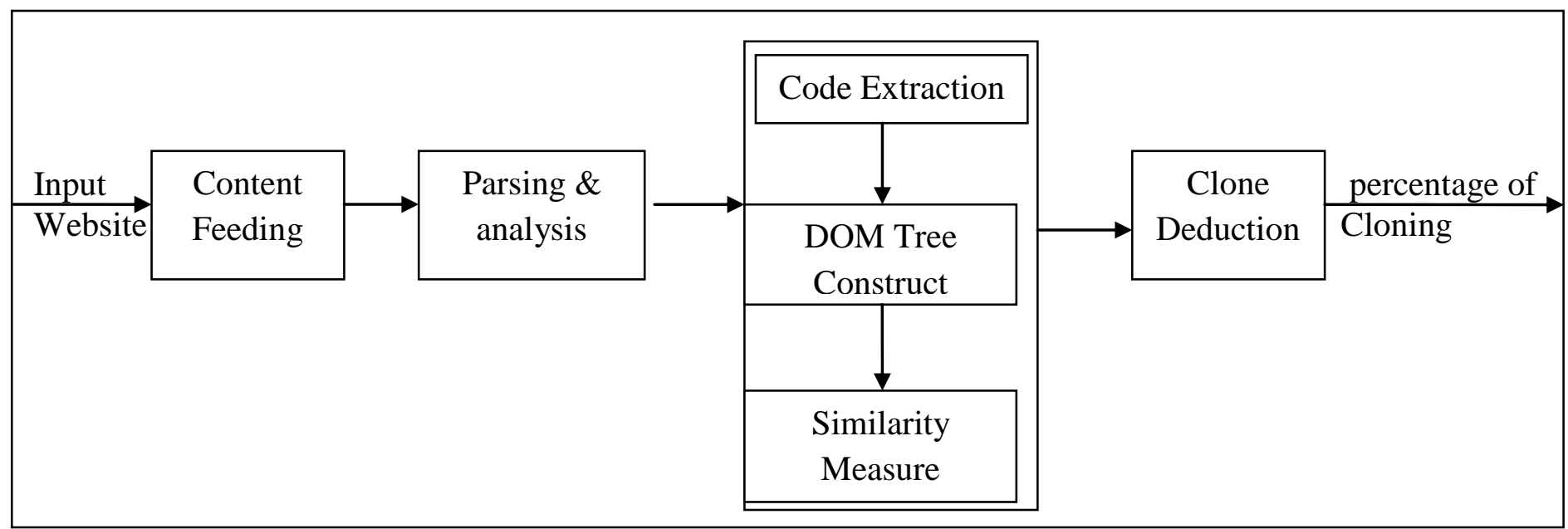


Step 5: Perform refactoring and Similarity measure using Levenshtein's approach.

Step 6: Detect the Clone from the code

The algorithm of the proposed methodology based on levenshtein distance measure is given below

\subsection{Description}

\subsubsection{Input and content extraction}

In the initial step, WebPages are read. The given input WebPages is transferred to the next phase to extract the contents. Web page are extracted one by one and the content (or) pieces of webpage code and extracted sequentially. Further these contents are forwarded to parse analysis [2].

\subsubsection{Parsing and analysis}

During this phase, the HTML parsing module accesses the HTML as tokens. It gives one token at a time, much as a file handler which gives one line at a time from a file. The HTML is tokenized from the input file as a string. The tokenize decodes the entities in attributes [35].

\subsubsection{Tree construction}

The tokenizes passes the output to construct tree. The data instances of the same type have the same path from the root in the DOM tree of the input page according to the page generation model. This method focuses on all levels of nodes. It starts from the root node <HTML>. It uses multiple string arguments approach to the first level child node [35].

\subsubsection{Similarity Measure}

The next level of the method is to computes the similarity measures using the levenshtein distance approach. It is based on matrix. A matrix is reserved to hold the distance between all prefix of the first string and all prefix of the second Afterwards computation is done on values of the matrix in a dynamic program. Fashion and them the distantness but the two full strings can be measure [36].

\subsubsection{Clone Detection}

The last step of the stage of the method is to detect the clone values from the outcome of the previous step. Clone detects values (\%) and clone index values are identified. The experimental results are discussed in the next section. Path from the root in the DOM tree of the input page according to the page generation model. This method focuses on all levels of nodes. It starts from the root node <HTML>. It uses multiple string arguments approach to the first level child node [4].

\section{EXPERIMENTAL RESULTS \& DISCUSSION}

The proposed refactoring techniques for clone detection have been implemented in $\mathrm{C \#}$ and experimental results are observed. The following sources shown in table 1 and table 2 are used for the experiments.

Table 1 : The HTML files analyzed in the experimental

\begin{tabular}{|c|l|c|}
\hline File ID & File Name & KB \\
\hline 1 & Index.html & 8.07 \\
\hline 2 & ISpecial list Imain frame.html & 0.411 \\
\hline 3 & ISpecial list ISpecial list.html & 1.75 \\
\hline 4 & ISpecial list text.html & 2.30 \\
\hline
\end{tabular}

\begin{tabular}{|c|c|c|}
\hline 5 & ISpecial list \title.html & 0.363 \\
\hline 6 & INovita \Brugaletta.html & 6.57 \\
\hline 7 & WNovita \CalendariotarNA.html & 10.6 \\
\hline 8 & Wovita \text.html & 3.30 \\
\hline 9 & \Title.html & 0.409 \\
\hline 10 & IForum Imain frame.html & 0.506 \\
\hline 11 & FForum Itaxt.html & 0.237 \\
\hline 12 & IForum Ititle.html & 0.4 \\
\hline 13 & ICommon frame left.html & 4.78 \\
\hline 14 & ICommon lbottom frame.html & 3.21 \\
\hline 15 & Main frame.html & 0.494 \\
\hline 16 & lirctc.html & 0.46 \\
\hline 17 & ljust dial.html & 0.58 \\
\hline 18 & IChisiamo Itext.html & 3.24 \\
\hline 19 & IChisiamo Ititle.html & 0.407 \\
\hline 20 & ICerca.html & 1.87 \\
\hline 21 & ICerca Imain frame.html & 0.501 \\
\hline 22 & ICerca Itext.html & 27.3 \\
\hline 23 & ICerca Ititle.html & 0.4 \\
\hline 24 & \Honda.html & 0.48 \\
\hline 25 & ISwift.html & 0.24 \\
\hline 26 & \TNEB.html & 0.20 \\
\hline 27 & Redbus.html & 0.44 \\
\hline 28 & INDTV.html & 0.90 \\
\hline 29 & Default.html & 0.96 \\
\hline 30 & ISample.html & 0.79 \\
\hline 31 & Waukri.html & 0.125 \\
\hline 32 & IVAT.html & 0.52 \\
\hline 33 & Live cricket.html & 0.269 \\
\hline 34 & Inaukri.html & 0.125 \\
\hline
\end{tabular}

Table 2 : Real time HTML files created by the volunteers

\begin{tabular}{|c|c|c|}
\hline File ID & File Name & KB \\
\hline 1 & $\backslash \mathrm{A} 1 . \mathrm{html}$ & 0.5 \\
\hline 2 & $\backslash \mathrm{A} 2 . \mathrm{html}$ & 0.2 \\
\hline 3 & $\backslash \mathrm{B} 1 . \mathrm{html}$ & 0.7 \\
\hline 4 & $\backslash \mathrm{B} 2 . \mathrm{html}$ & 0.4 \\
\hline 5 & $\backslash \mathrm{C} 1 . \mathrm{html}$ & 0.2 \\
\hline 6 & $\backslash \mathrm{C} 2 . \mathrm{html}$ & 0.3 \\
\hline 7 & $\backslash \mathrm{C} 2 . \mathrm{html}$ & 0.1 \\
\hline 8 & $\backslash \mathrm{C} 3 . \mathrm{html}$ & 0.20 \\
\hline
\end{tabular}




\begin{tabular}{|c|c|c|}
\hline 9 & L1.html & 0.12 \\
\hline 10 & L2.html & 0.10 \\
\hline 11 & LE1.html & 0.7 \\
\hline 12 & LE2.html & 0.4 \\
\hline
\end{tabular}

The results of two files from the above mentioned table are shown below in table 3. For instance the results of two files namely Honda.html and SuzukiSwift.html are listed in table1. It lists the tag index and clone detection value for both files which are taken from open sources as mention in table 1 .

Table 3 : Tag index and clone detection value for two files (Honda, Swift)

\begin{tabular}{|c|c|c|c|}
\hline \multicolumn{2}{|c|}{ F1 : Honda.html } & \multicolumn{2}{c|}{ F2 : Swift.html } \\
\hline $\begin{array}{c}\text { Tags } \\
\text { index }\end{array}$ & $\begin{array}{c}\text { Clone } \\
\text { detection } \\
\text { value }\end{array}$ & $\begin{array}{c}\text { Tags } \\
\text { index }\end{array}$ & $\begin{array}{c}\text { Clone } \\
\text { detection } \\
\text { value }\end{array}$ \\
\hline doc type & 1 & doc type & 1 \\
\hline Html & 1 & html & 1 \\
\hline Head & 1 & head & 1 \\
\hline Meta & 5 & mea & 2 \\
\hline Title & 1 & File & 1 \\
\hline Script & 55 & Link & 12 \\
\hline Script & 55 & Link & 1 \\
\hline Link & 8 & Body & 1 \\
\hline Style & 3 & Div & 12 \\
\hline Body & 1 & Ul & 6 \\
\hline Form & 1 & Li & 48 \\
\hline Div & 153 & A & 59 \\
\hline Input & 44 & A & 59 \\
\hline Input & 43 & Sript & 14 \\
\hline Div & 153 & Script & 10 \\
\hline Input & 44 & Ins & 10 \\
\hline Input & 43 & Ins & 5 \\
\hline A & 288 & Fname & 5 \\
\hline Img & 153 & H1 & 1 \\
\hline Select & 1 & H2 & 2 \\
\hline Option & 1 & P & 32 \\
\hline Strong & 46 & P & 31 \\
\hline Span & 74 & Img & 2 \\
\hline Table & 30 & B & 11 \\
\hline
\end{tabular}

\begin{tabular}{|c|c|c|c|}
\hline Tbody & 30 & B & 10 \\
\hline $\mathrm{Tr}$ & 78 & H3 & 9 \\
\hline $\mathrm{Td}$ & 166 & Strong & 2 \\
\hline $\mathrm{Br}$ & 89 & $\mathrm{Br}$ & 42 \\
\hline Ul & 38 & $\mathrm{Br}$ & 30 \\
\hline $\mathrm{Li}$ & 204 & Table & 1 \\
\hline $\mathrm{Li}$ & 73 & Tbody & 1 \\
\hline Form & 1 & $\operatorname{Tr}$ & 6 \\
\hline Div & 153 & $\mathrm{Td}$ & 23 \\
\hline Input & 44 & $\mathrm{Td}$ & 1 \\
\hline Input & 43 & Ui & 1 \\
\hline A & 288 & File & 6 \\
\hline Img & 153 & Small & 8 \\
\hline Select & 1 & Small & 4 \\
\hline Option & 1 & Form & 1 \\
\hline Strong & 46 & Input & 6 \\
\hline Table & 30 & Lable & 2 \\
\hline Tbody & 30 & Lable & 1 \\
\hline $\mathrm{Tr}$ & 78 & Text area & 1 \\
\hline $\mathrm{Td}$ & 166 & No script & 1 \\
\hline $\mathrm{H} 2$ & 1 & - & - \\
\hline Em & 1 & - & - \\
\hline Em & 1 & - & - \\
\hline Font & 2 & - & - \\
\hline Font & 2 & - & - \\
\hline $\mathrm{H} 4$ & 1 & - & - \\
\hline Embed & 1 & - & - \\
\hline B & 2 & - & - \\
\hline B & 1 & - & - \\
\hline Map & 1 & - & - \\
\hline Area & 3 & - & - \\
\hline $\mathrm{H} 3$ & 3 & - & - \\
\hline
\end{tabular}

Fig. 2 Visualizes clone detection value of the above mentioned files. From the result, it is observed and calculated the clone detection value. This result shows the html tags and index value of first file (Honda.html) and second file (Swift.html). About $26.1 \%$ of code clones are identified from the two files. 


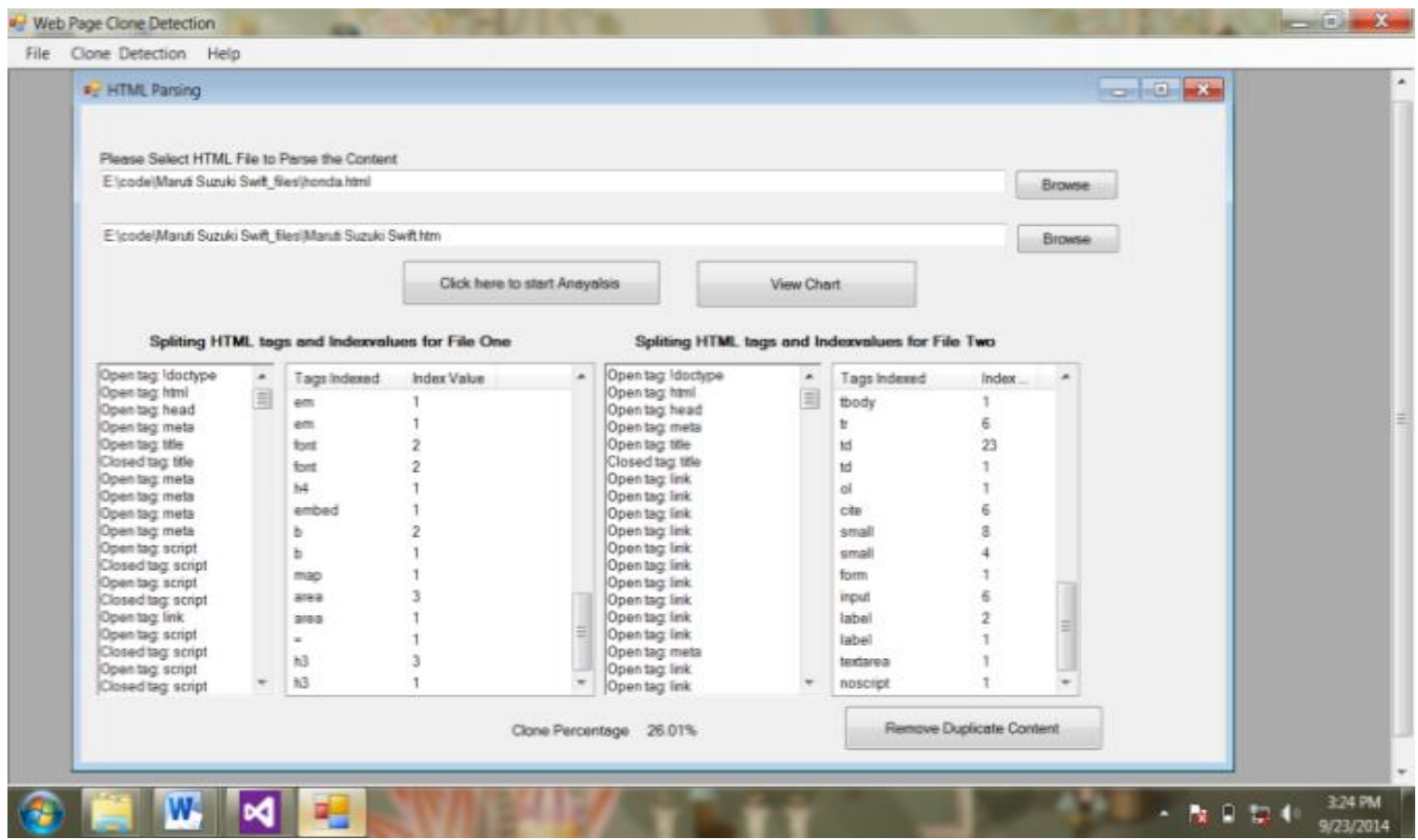

Figure 2: Comparison of clone detection value of two files (F1 : Honda, F2 : Swift).

In Fig. 3 The upper portion of the screen shows the individual clone detection of F1 and F2. Lower portion of the screen shows the comparison of clone detection value of two files.

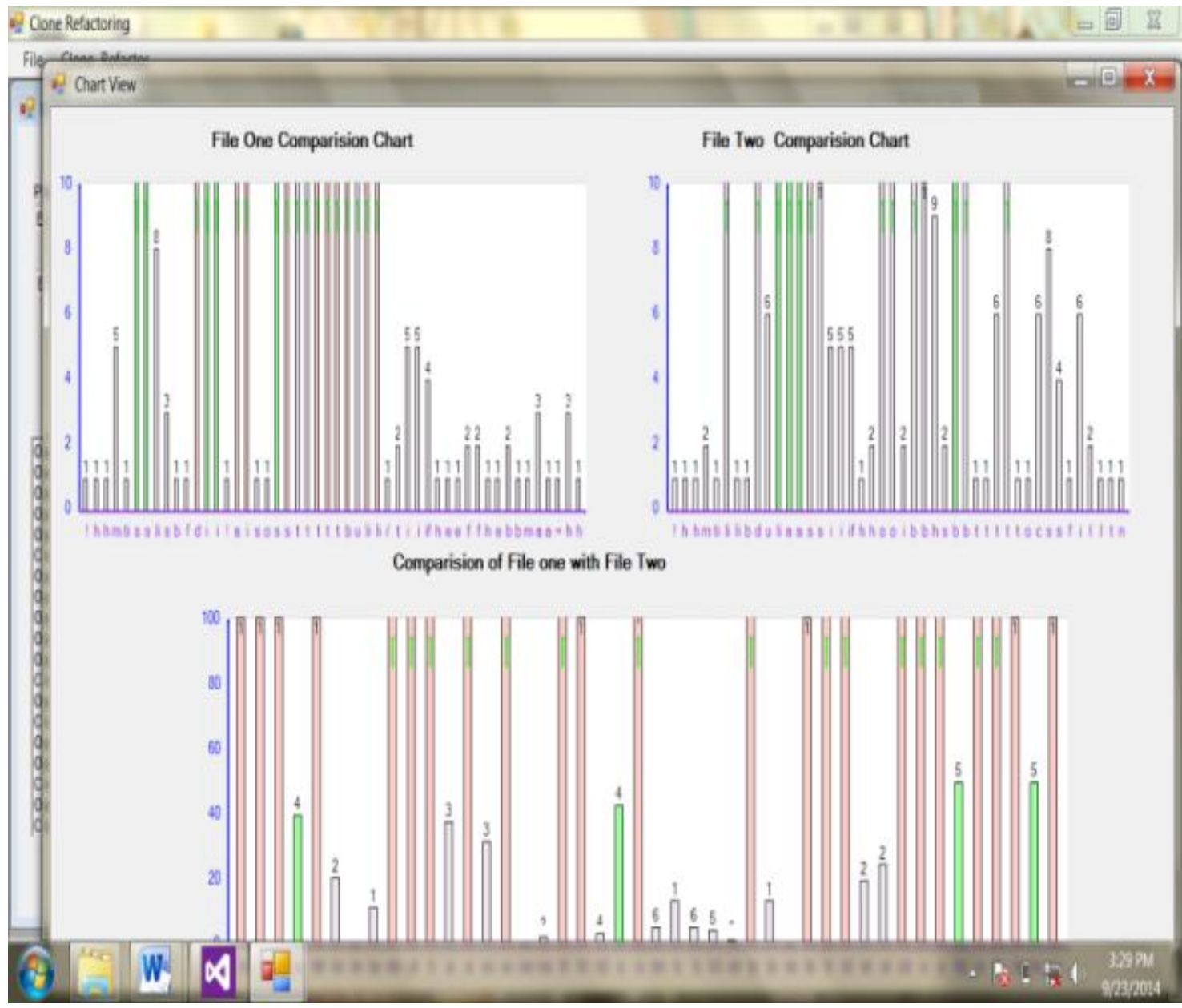

Figure 3: Comparison chart of clone detection on individual values (html tags / and index values). 
Table 4: Tag index and clone detection value for two files (TNEB, Redbus)

\begin{tabular}{|c|c|c|c|}
\hline \multicolumn{2}{|l|}{ F3: TNEB.html } & \multicolumn{2}{l|}{ F4 Redbus.html } \\
\hline $\begin{array}{c}\text { Tags } \\
\text { index }\end{array}$ & $\begin{array}{c}\text { Clone } \\
\text { deduction } \\
\text { value }\end{array}$ & $\begin{array}{c}\text { Tags } \\
\text { index }\end{array}$ & $\begin{array}{c}\text { Clone } \\
\text { value }\end{array}$ \\
\hline doc type & 1 & doc type & 1 \\
\hline html & 1 & Html & 1 \\
\hline head & 1 & Head & 1 \\
\hline Meta & 1 & Meta & 2 \\
\hline Link & 2 & Title & 1 \\
\hline Link & 2 & Link & 4 \\
\hline Script & 4 & Link & 3 \\
\hline Title & 1 & Script & 21 \\
\hline Body & 1 & Script & 18 \\
\hline Table & 1 & Body & 1 \\
\hline Tbody & 4 & Div & 100 \\
\hline Tr & 18 & Header & 1 \\
\hline Td & 53 & Ul & 6 \\
\hline Title & 1 & Li & 73 \\
\hline Body & 1 & A & 87 \\
\hline Table & 4 & A & 85 \\
\hline Tbody & 4 & Span & 77 \\
\hline Tr & 18 & Span & 21 \\
\hline Td & 53 & Img & 6 \\
\hline
\end{tabular}

\begin{tabular}{|c|c|c|c|}
\hline Img & 9 & $\mathrm{Br}$ & 1 \\
\hline $\mathrm{P}$ & 2 & $\mathrm{H} 3$ & 1 \\
\hline A & 44 & Section & 2 \\
\hline Font & 1 & H1 & 1 \\
\hline Div & 23 & Label & 15 \\
\hline Span & 27 & Input & 13 \\
\hline Span & 21 & Input & 11 \\
\hline Form & 1 & Button & 9 \\
\hline $\mathrm{Br}$ & 3 & Aside & 1 \\
\hline $\mathrm{Br}$ & 2 & Footer & 1 \\
\hline Thead & 1 & H6 & 1 \\
\hline Th & 6 & Sup & 1 \\
\hline Th & 1 & $\mathrm{P}$ & 1 \\
\hline Input & 3 & Fname & 4 \\
\hline Input & 1 & Noscript & 2 \\
\hline B & 1 & Noscript & 1 \\
\hline- & - & Table & 4 \\
\hline- & - & Tbody & 4 \\
\hline- & - & $\operatorname{Tr}$ & 32 \\
\hline - & - & $\mathrm{Td}$ & 179 \\
\hline- & - & $\mathrm{Td}$ & 178 \\
\hline - & - & Th & 27 \\
\hline - & - & Th & 1 \\
\hline - & - & $\mathrm{H} 2$ & 2 \\
\hline
\end{tabular}

Fig. 4 Visualizes clone detection value of the above mentioned files. From the result, the clone detection value is calculated. This result shows the html tags and index value of F3 and F4. About $21.81 \%$ of code clones are identified in between two files (TNEB.html, Redbus.html).

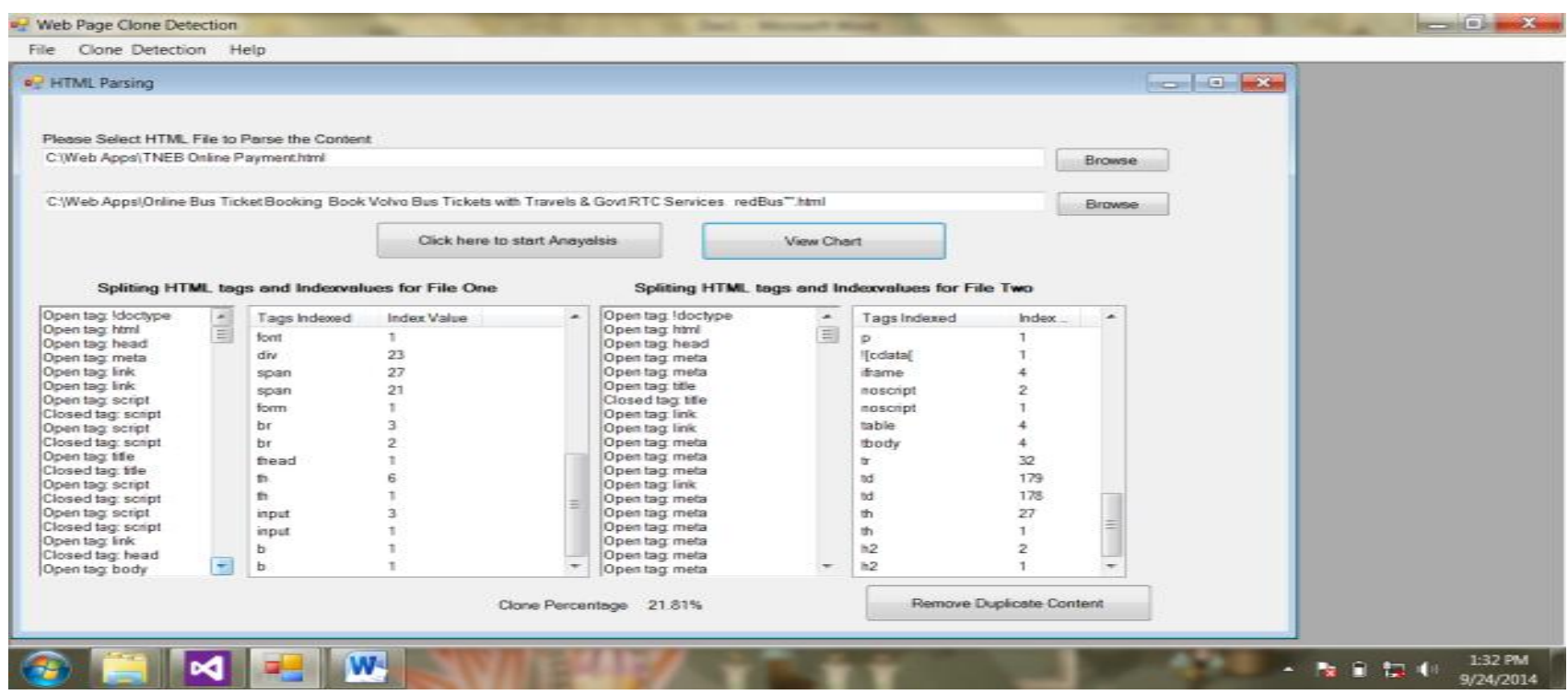

Figure 4 : Comparison of clone detection value of two files (F3 : TNEB, F4 : Redbus). 
In fig. 5 the upper portion of the chart shows the individual clone detection of F3 and F4. The lower portion of the represents the comparison of clone detection value of two files. This chart shows the individual clone detection value of F3 and F4. The below chart shows the comparison of clone detection value of two files.

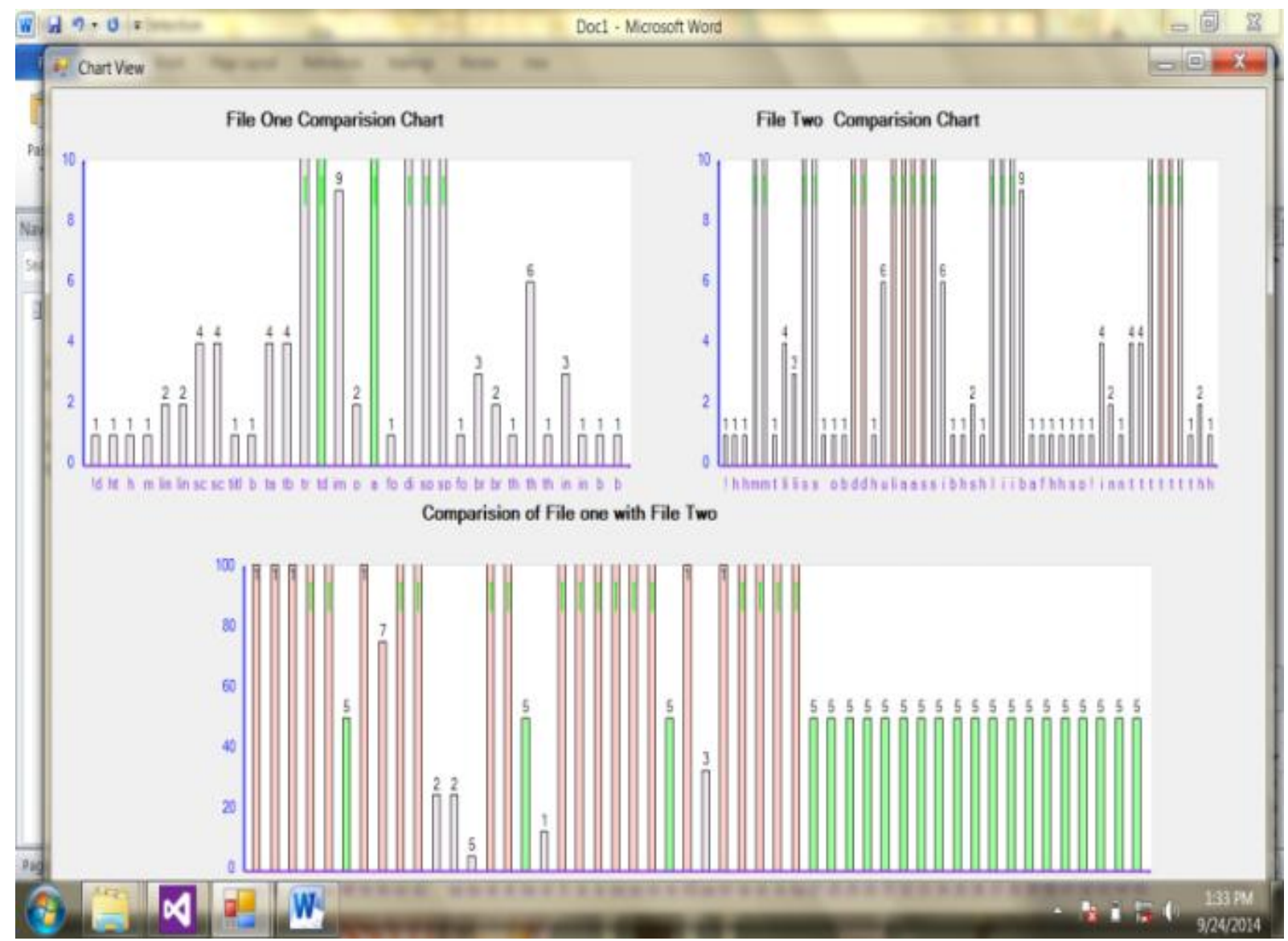

Figure 5: Comparison chart of clone detection on individual values (html tags / and index values).

Table 5: Tag index and clone detection value for two files (A1.html , A2.html) created by voluntaries.

\begin{tabular}{|c|c|c|c|}
\hline \multicolumn{2}{|c|}{ F5:A1.html } & \multicolumn{2}{c|}{ F6 : A2.html } \\
\hline $\begin{array}{c}\text { Tags } \\
\text { index }\end{array}$ & $\begin{array}{c}\text { Clone deduction } \\
\text { value }\end{array}$ & $\begin{array}{c}\text { Tags } \\
\text { index }\end{array}$ & $\begin{array}{c}\text { Clone } \\
\text { value }\end{array}$ \\
\hline Html & 1 & Html & 1 \\
\hline Head & 1 & Head & 1 \\
\hline Title & 1 & Title & 1 \\
\hline Body & 1 & Body & 1 \\
\hline H1 & 1 & H1 & 1 \\
\hline H2 & 1 & H2 & 1 \\
\hline
\end{tabular}

\begin{tabular}{|c|c|c|c|}
\hline H2 & 1 & H2 & 1 \\
\hline Left & 1 & Left & 1 \\
\hline U1 & 5 & A & 1 \\
\hline Li & 4 & B & 5 \\
\hline A & 1 & B & 5 \\
\hline B & 1 & B & 4 \\
\hline B & 1 & - & - \\
\hline P & 4 & - & - \\
\hline P & 1 & - & - \\
\hline Div & 1 & - & - \\
\hline
\end{tabular}

Fig. 6 Visualizes clone detection value of the above mentioned files that the result shows the html tags and index value of F5 and F6. About $19.35 \%$ of code clones are identified between two files (A1.html, A2.html). 


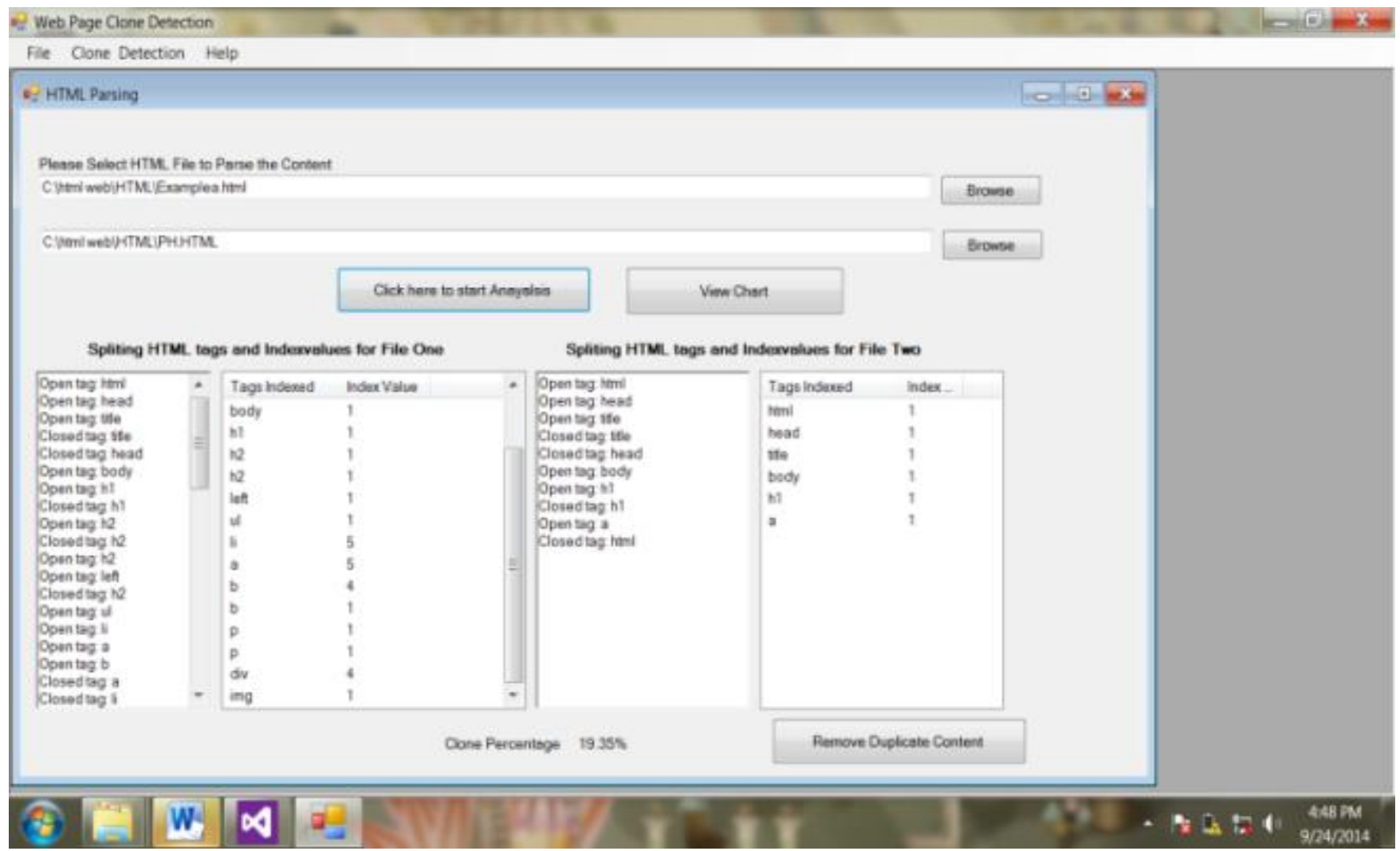

Figure 6: Comparison of clone detection value of two files (F5: A1, F6 : A1).

In fig. 7, the upper portion of the chart shows the individual clone detection of F5 and F6. The lower portion of the chart compares the clone detection value of two files.

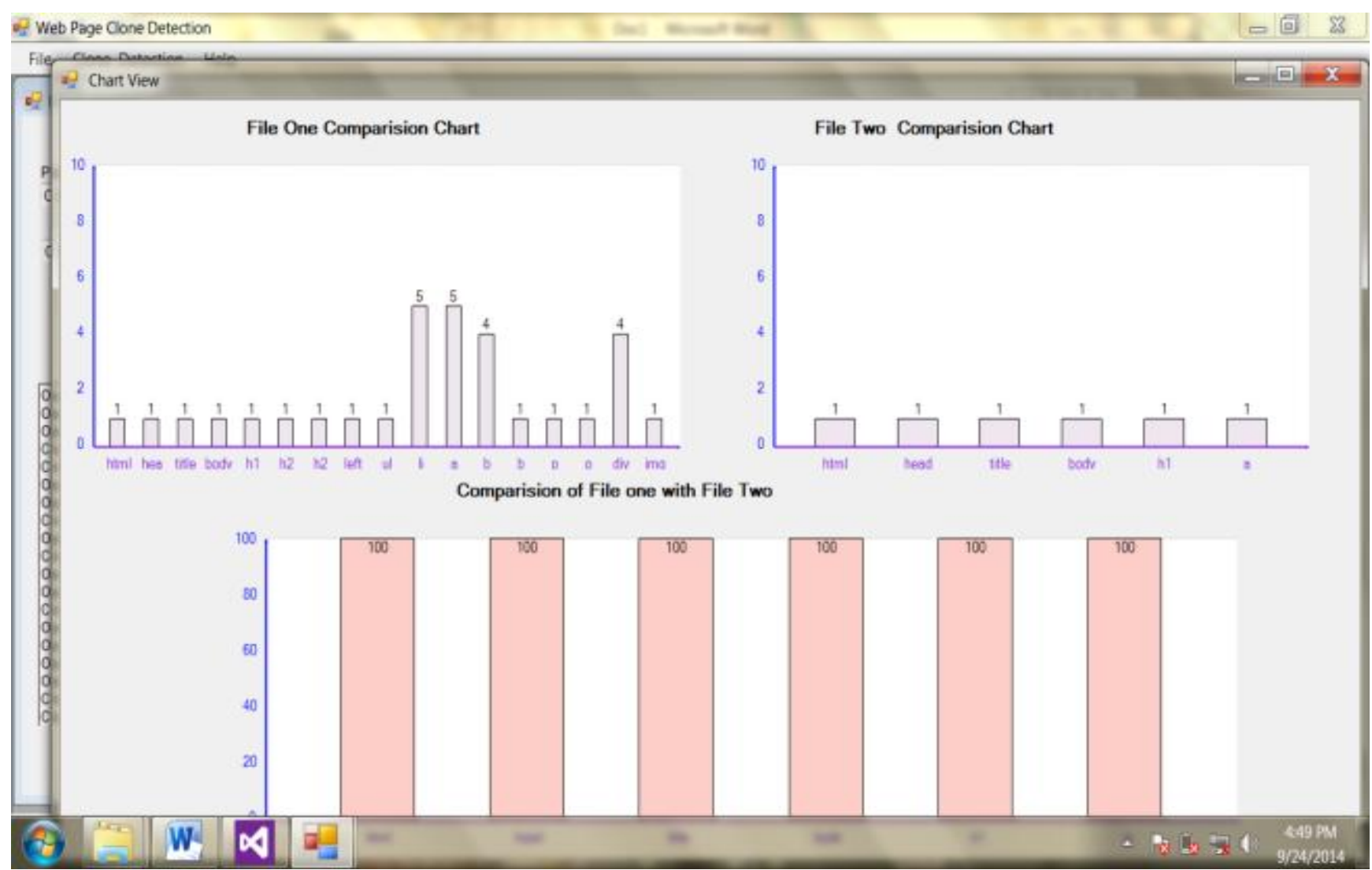

Figure 7: Comparison chart of clone detection on individual values (html tags / and index values). 


\subsection{Performance Measure}

The performance of the proposed methods is based on clone percentage and also time taken to detect the clone. Table 6 lists the two measures for some files.

Table 6: Performance measure on clone percentage

\begin{tabular}{|l|l|c|c|}
\hline \multicolumn{2}{|c|}{} & \multicolumn{2}{c|}{ Clone percent (\%) } \\
\cline { 3 - 4 } \multicolumn{2}{|c|}{ Name of the webpage(s) } & $\begin{array}{l}\text { Static } \\
\text { web } \\
\text { pages }\end{array}$ & $\begin{array}{l}\text { Dynamic } \\
\text { web } \\
\text { pages }\end{array}$ \\
\hline Default.html & Net carry .html & 100 & 40 \\
\hline Swift.html & Net carry .html & 33 & 27 \\
\hline Search.html & Compile.asp & 24 & 46 \\
\hline Code.html & Sample.asp & 29 & 70 \\
\hline
\end{tabular}

Chart describes fig. 8 clone detection percentage of static and dynamic web pages.

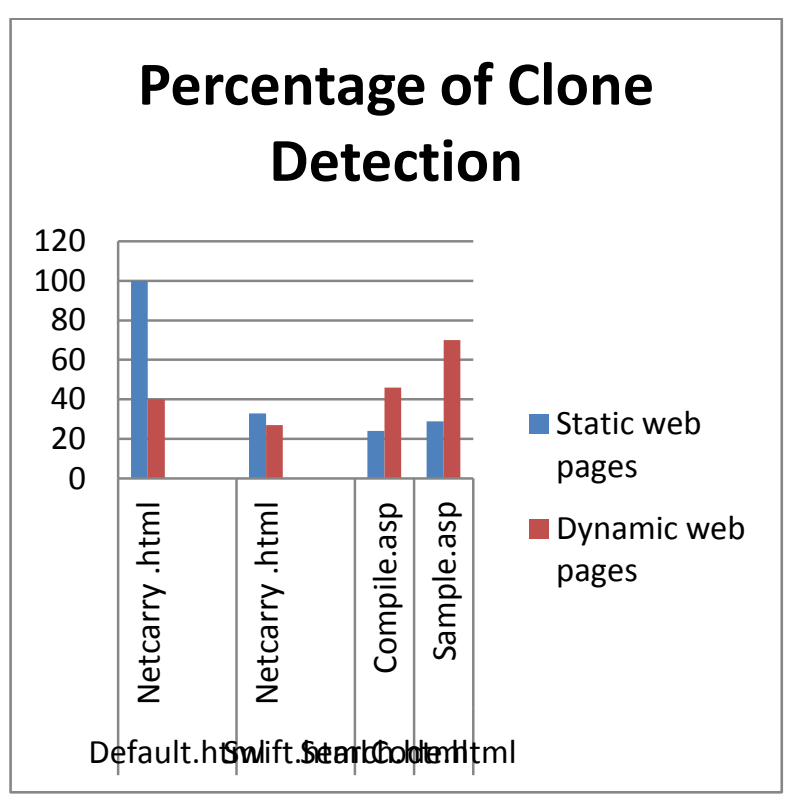

Figure 8: This chart measure comparison of clone percentage.

Table.7: Performance measure to time taken of clone detection

\begin{tabular}{|l|l|c|}
\hline \multicolumn{2}{|c|}{ Name of the webpage(s) } & $\begin{array}{c}\text { Clone } \\
\text { detection time } \\
\text { (in seconds) }\end{array}$ \\
\hline Default.html & Net carry .html & 0.55 \\
\hline Swift.html & Net carry .html & 0.12 \\
\hline Search.html & Compile.asp & 0.06 \\
\hline Code.html & Sample.asp & 0.05 \\
\hline
\end{tabular}

Table 7 and Fig. 9 indicate the time measure of clone detection in open source web pages.

\section{Clone detection time (in seconds)}

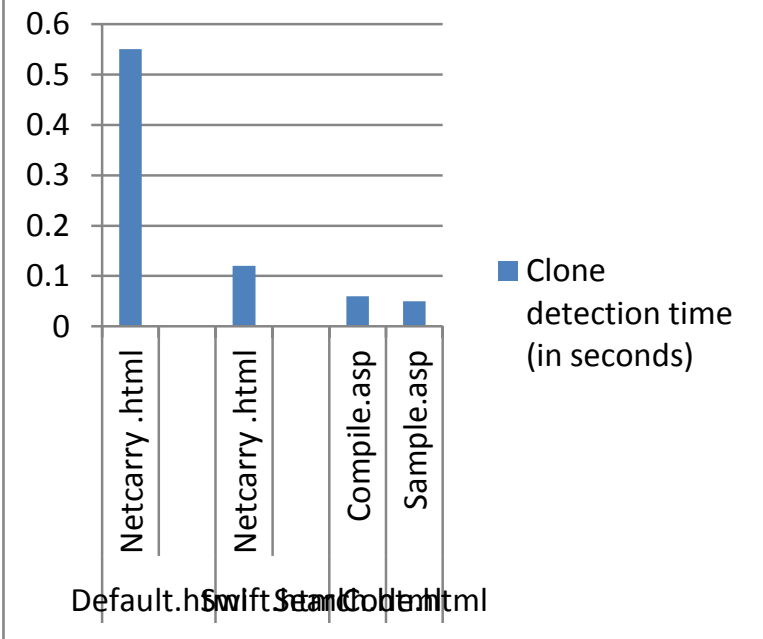

Figure 9: This chart measures clone detection time (In seconds).

Table 8: Performance measure on clone percentage

\begin{tabular}{|l|l|c|c|}
\hline \multirow{2}{*}{\multicolumn{2}{|c|}{}} & \multicolumn{2}{c|}{ Clone percent (\%) } \\
\cline { 3 - 4 } \multicolumn{2}{|c|}{ Name of the webpage(s) } & $\begin{array}{c}\text { Static } \\
\text { web pages }\end{array}$ & $\begin{array}{c}\text { Dynamic } \\
\text { web pages }\end{array}$ \\
\hline B1.html & A1.html & 49 & 80 \\
\hline B2.html & A2.html & 67 & 55 \\
\hline VAT.html & Compile.asp & 58 & 93 \\
\hline C1.html & Sample.asp & 49 & 35 \\
\hline
\end{tabular}

Table 8, 9 and Fig. 10, 11 give information about the clone detection percentage of static and dynamic web page.

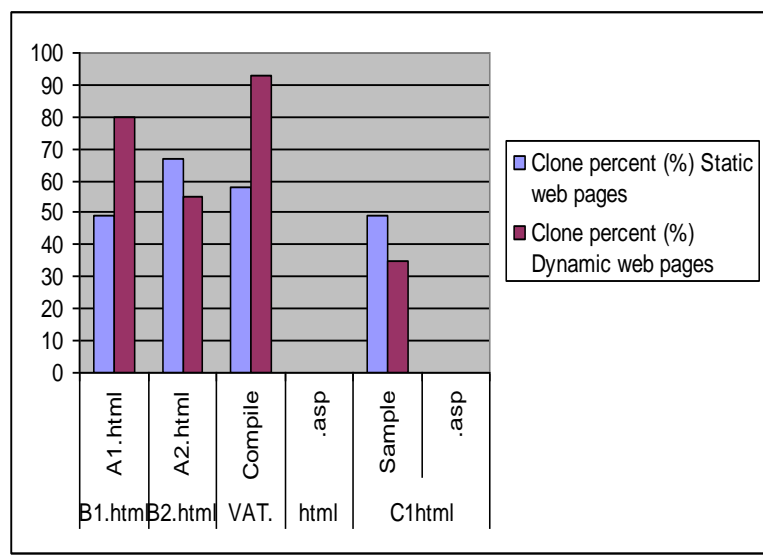

Figure 10: This chart measure comparison of clone percentage. 
Table 9 : Performance measure to time taken of clone detection

\begin{tabular}{|l|l|c|}
\hline \multicolumn{2}{|l|}{ Name of the webpage(s) } & $\begin{array}{c}\text { Clone detection } \\
\text { time } \\
\text { (in seconds) }\end{array}$ \\
\hline B1.html & A1.html & 0.58 \\
\hline B2.html & A2.html & 0.10 \\
\hline VAT.html & Compile.asp & 0.05 \\
\hline C1.html & Sample.asp & 0.03 \\
\hline
\end{tabular}

Fig. 11 gives information about the clone detection percentage of static and dynamic web page.

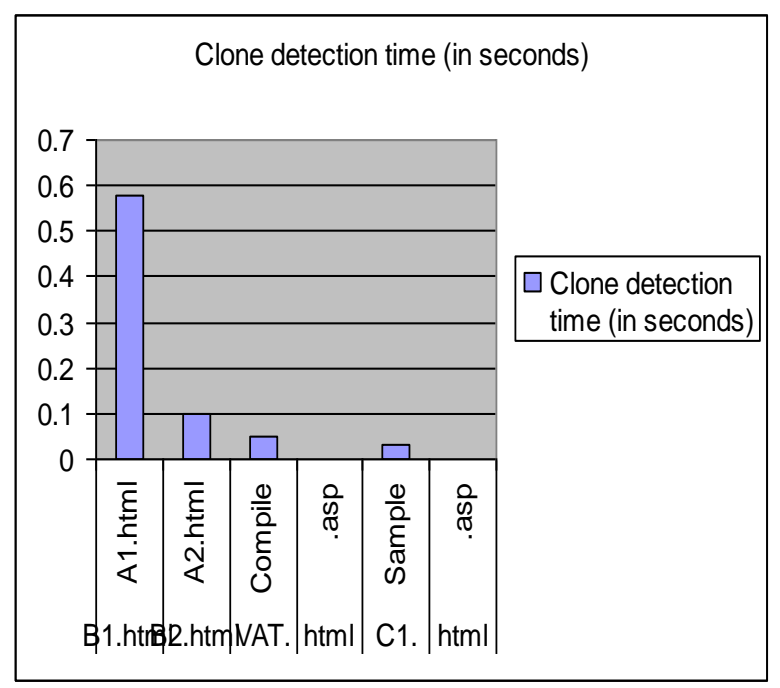

Figure 11: This chart measures clone detection time (In seconds).

\section{CONCLUSION}

Code clone detection is an art of detecting the content similarity between the programs or WebPages. An attempt is made to design a method called "SD Code Clone Detection" for both static and dynamic WebPages. It is based on levenshtein's approach. This method comprises some steps like, parsing \& analysis, tree construction, code similarity measure and clone detection. Experiments are carried out with open source websites and WebPages created by some volunteers. Experimental results are recorded and are showing the better detection rate. Future research on Web data extraction focuses on comparing the contents appearing on the page as well as the code to measure the standard and originality of the web page. However, they are redesigned or applied in a different sequence and scenario to solve key issues in page-level data extraction and comparison to the code of web site and its contents to find the fake and the real. The System can also be enhanced work to detect the script injection and projected towards the detection of malwares attached to web pages that harms the user's machine and acts as a spy ware and sends the information of the end user to the attacker. These systems are still in research to prevent the attackers. It is planned to exploit the results of the clone mining method to support web application reengineering activities.

\section{REFERENCES}

[1] Aversano, L., Canfora, G., De Lucia, A., and Gallucci, P., 2001. Web Site Reuse: Cloning and Adapting. Proc. Of 3rd International Workshop on Web Site Evolution, Florence, Italy, IEEE CS Press, pp. 107-111.

[2] Chang C.-H. and S.-C. Lui, "IEPAD: Information Extraction Based on Pattern Discovery," Proc. Int'l Conf. World Wide Web (WWW-10), pp. 223-231, 2001.

[3] Cloning http://msdn.microsoft.com/enus/library/hh205279.aspx

[4] De Lucia, A., Scanniello, G., and Tortora, G., 2004."Identifying Clones in Dynamic Web Sites Using Similarity Thresholds," Proc. Intl. Conf. on Enterprise Information Systems (ICEIS'04), pp.391-396.

[5] Daniel B, D. Dig, K. Garcia, and D. Marinov, "Automated Testing of Refactoring Engines," Proc. Sixth Joint Meeting European Software Eng. Conf. and ACM SIGSOFT Symp. The Foundations of Software Eng., pp. 185-194, 2007.

[6] Dig D, and R. Johnson, "The Role of Refactorings in API Evolution," Proc. 21st IEEE Int'l Conf. Software Maintenance,pp. 389-398, 2005.

[7] Eclipse.org, "Eclipse Project,"at http://www.eclipse.org, 2011.

[8] EmbarcaderoTechnologies, “JBuilder," http://www.codegear.com/br/products/jbuilder, 2011.

[9] "JDT Core Component," Eclipse.org, http://www.eclipse.org/ jdt/core/, 2011.

[10] Fowler M., Refactoring: Improving the Design of Existing Code. Addison-Wesley Longman Publishing Co., 1999.

[11] Goodenough J.B. and S.L. Gerhart, "Toward a Theory of Test Data Selection," SIGPLAN Notes, vol. 10, pp. 493510, Apr. 1975.

[12] Gligoric M, T. Gvero, V. Jagannath, S. Khurshid, V. Kuncak, and D. Marinov, "Test Generation through Programming in UDITA," Proc. 32nd Int'l Conf. Software Eng., vol. 1, pp. 225-234, 2010.

[13] Hoffman D.M, D. Ly-Gagnon, P. Strooper, and H.-Y. Wang, "Grammar-Based Test Generation with YouGen," Software: Prac- tice and Experience, vol. 41, pp. 427447, Apr. 2011.

[14] Jackson D., I. Schechter, and H. Shlyahter, "Alcoa: The Alloy Constraint Analyzer," Proc. 22nd Int'1 Conf. Software Eng., pp. 730-733, 2000.

[15] Jin W, A. Orso, and T. Xie, "Automated Behavioral Regression Testing," Proc. 23rd Int'l Conf. Software Testing, Verification and Validation, pp. 137-146, 2010.

[16] Kushmerick, D. Weld, and R. Doorenbos, "Wrapper Induction for Information Extraction," Proc. 15th Int'1 Joint Conf. Artificial Intelligence (IJCAI), pp. 729-735, 1997.

[17] Muslea I., S. Minton, and C. Knoblock, "A Hierarchical Approach to Wrapper Induction," Proc. Third Int'l Conf. Autonomous Agents (AA '99), 1999. 
[18] Mens T. and T. Tourwe', "A Survey of Software Refactoring," IEEE Trans. Software Eng., vol. 30, no. 2, pp. 126-139, Feb. 2004.

[19] Demeyer. S, Mens T, and D. Janssens, "Formalising Behaviour Preserving Program Transformations," Proc. First Int'l Conf. Graph Transformation, pp. 286-301, 2002.

[20] Marinov D and S. Khurshid, "TestEra: A Novel Framework for Automated Testing of Java Programs," Proc. IEEE 16th Int'l Conf. Automated Software Eng., pp. 22-34, 2001

[21] Opdyke W.F, "Refactoring Object-Oriented Frameworks," $\mathrm{PhD}$ dissertation, Univ. of Illinois at Urbana-Champaign, 1992.

[22] Overbey J.L and R.E. Johnson, "Differential Precondition Check-ing: A Lightweight, Reusable Analysis for Refactoring Tools," Proc. 26th IEEE/ACM Int'l Conf. Automated Software Eng., pp. 303-312,

[23] Refactoring http://www.informit.com/articles/article.aspx

[24] Simon K and G. Lausen, "ViPER: Augmenting Automatic Information Extraction with Visual Perceptions," Proc. Int'l Conf. Information and Knowledge Management (CIKM), 2005.

[25] Sun Microsystems, "NetBeans IDE," http://www.netbeans.org/,2011.

[26] Scha fer M, M. Verbaere, T. Ekman, and O. Moor, "Stepping Stones over the Refactoring Rubicon," Proc. 23rd European Conf. Object-Oriented Programming, pp. 369-393, 2009.

[27] Moor O. de, Scha"fer $M$ and "Specifying and Implementing Refactorings," Proc. 25th ACM Int'l Conf. Object-Oriented Programming, Systems, Languages, and Applications, pp. 286-301, 2010.
[28] Ekman T. Scha "fer M, and O. de Moor, "Challenge Proposal: Verification of Refactorings," Proc. Third Workshop Programming Languages Meets Program Verification, pp. 67-72, 2008.

[29] Gheyi. R Soares G, D. Serey, and T. Massoni, "Making Program Refactoring Safer," IEEE Software, vol. 27, no. 4, pp. 52-57, July/ Aug. 2010.

[30] Mongiovi M., Soares G, and R. Gheyi, "Identifying Overly Strong Conditions in Refactoring Implementations," Proc. Conf. Software Maintenance, pp. 173-182, Sept. 2011

[31] Silva L, A. Sampaio, and Z. Liu, "Laws of ObjectOrientation with Reference Semantics," Proc. Sixth IEEE Int'l Conf. Software Eng. And Formal Methods, pp. 217 226, 2008.

[32] Moor O. de, Scha fer M, T. Ekman, and "Sound and Extensible Renaming for Java," Proc. 23rd ACM SIGPLAN Conf. Object Oriented Programming, Systems, Languages, and Applications, pp. 277-294, 2008.

[33] Tokuda L and D. Batory, "Evolving Object-Oriented Designs with Refactoring," Automated Software Eng., vol. 8, pp. 89-120, Jan.2001

[34] Sourcemaking http://sourcemaking.com/refactoring/introduction-torefactoring

[35] DOM Tree Algorithm :http://dbs.snu.ac.kr/papers/xsym09.pdf.

[36] Levenshtein Edit Distance Algorithm: http://www.levenshtein.net/ levenshtein measure http://en.wikibooks.org/wiki/Algorithm_Implementation/ Strings/Levenshtein_distance. 